\title{
Revisão sobre modelos volumétricos empregados em povoamentos florestais brasileiros
}

\author{
Valdir Carlos Lima de Andrade ${ }^{1 *}$ Rafaella da Costa Gama ${ }^{1}$ David Lucas Camargo Vieira Terra ${ }^{1}$ \\ ${ }^{1}$ Universidade Federal do Tocantins, Campus Gurupi, Chácara 69-72 - Rua Badejos, Lote 7, s/n - Jardim Cervilha, Gurupi - TO, 77404-970 \\ *Author for correspondence: vclandrade@ hotmail.com \\ Received: September 2018 / Accepted: December 2018 / Published: March 2019
}

\section{Resumo}

Uma das informações mais importantes no planejamento florestal é a quantificação do volume de madeira. Por isso, os objetivos deste artigo de revisão foram: identificar os procedimentos e critérios estatísticos mais usuais para o ajuste de modelos volumétricos, os tipos de povoamento estudados, os modelos mais utilizados, assim como o cenário geográfico dos estudos desse importante assunto. Os gêneros mais estudados são Eucalyptus e Pinus. Com relação à fonte de dados, mais da metade (52,5\%) está concentrado em quatro estados brasileiros: Minas Gerais, Pará, Mato Grosso e Bahia. O método mais empregada para obtenção do volume rigoroso das árvores é a de Smalian. O modelo de Shumacher-Hall e de Spurr são os mais usuais. Contudo, destaca-se a importância de se testar vários modelos, visto que, para cada caso, outros modelos podem obter melhores resultados. Os critérios estatísticos mais utilizados são: coeficiente de determinação ajustado, erro padrão da estimativa e análise gráfica dos resíduos.

Palavras-chaves: Análise de regressão; dendrometria; cubagem rigorosa.

\begin{abstract}
One of the most important information in forest planning is to quantify the volume of wood. Thus, the objectives of this review article were to identify the procedures and most common statistical criteria for the adjustment of volumetric models, types of studied population, the most widely used models, as well as the geographic setting of the study of this important subject. The most studied genera are Eucalyptus and Pinus. With respect to the data source, more than half $(52,5 \%)$ is concentrated in five Brazilian states: Minas Gerais, Pará, Mato Grosso and Bahia. The most commonly used formula for obtaining accurate volume of trees is the Smalian. The model of Schumacher-Hall and Spurr are the most common. However, it highlights the importance of testing several other models, since in each case other models can best settings. The most commonly used statistical criteria are: adjusted coefficient, standard error of the estimate and graphical analysis of waste.
\end{abstract}

Keywords: Regression analysis; dendrometry; strict cubing.

\section{Introdução}

$\mathrm{O}$ volume de madeira, em estoque nos povoamentos florestais, representa uma das informações de maior relevância para o planejamento da produção florestal. É imprescindível para a realização de planos de manejo das florestas (Leite e Andrade 2002). Para quantificar esse volume, realiza-se um inventário florestal que consiste na inferência da população a partir da medição de parte da mesma. Neste caso, exige-se obter o volume por meio de equações volumétricas, preferencialmente, de dupla entrada.

Antes do desenvolvimento dos computadores, as equações volumétricas se davam pela elaboração manual de tabelas contendo os volumes estimados de cada árvore de acordo com as variáveis utilizadas. Esse tema é abordado como: "Tabelas de volume" (Paula Neto et al. 1983), sendo que, a mais antiga, foi elaborada na segunda metade do século XVIII. Segundo Spurr (1952), foi Henrich Cotta que publicou, em 1804 e 1817, as primeiras tabelas de volume onde se apresentou o conceito do volume de uma árvore ser dependente da altura, diâmetro e forma do tronco da mesma.

No Brasil, as primeiras tabelas de volume foram feitas a partir da década de 1960, conforme constam em: Andrade (1961), Heinsdjk (1965), Hosokawa e Kajiwa (1971), Veiga (1972), Siqueira (1977) e Machado (1979). Portanto, há quase 60 anos, muitas equações volumétricas já foram desenvolvidas para povoamentos florestais brasileiros.

Embora haja eficiência de algumas equações de volume, estas nem sempre se ajustam a todas as espécies e condições dos povoamentos florestais. Por isso, deve-se testar várias equações para selecionar a mais apropriada à condição de uso (Thomas et al. 2006; Soares et al. 2011; Santos et al. 2012; Melo et al. 2013). Dessa maneira, dezenas de modelos estatísticos foram testados, assim como, diferentes critérios foram utilizados para a seleção da melhor equação.

Considerando o exposto, os objetivos deste artigo de revisão foram: identificar os procedimentos e critérios estatísticos mais usuais na avaliação de modelos volumétricos, os tipos de povoamentos florestais estudados, os modelos volumétricos mais utilizados, assim como, o cenário geográfico dos estudos desse importante assunto da mensuração florestal.

\section{Caracterização do estudo}

Este trabalho foi desenvolvido por meio da análise de artigos científicos publicados sobre modelos volumétricos no Brasil, com enfoque nos trabalhos que avaliaram modelos volumétricos e não somente os utilizaram. Tais artigos, foram selecionados de forma aleatória através do mecanismo de busca "www.google.com". As palavras chaves principais utilizadas foram: modelos volumétricos, tabelas de volume, volumetria e modelagem volumétrica, equações volumétricas, volume eucalipto, volume pinus.

É importante ressaltar, que além do mecanismo "www.google.com", também, foram utilizadas as referências destacadas nos artigos encontrados. Também, os dados coletados através dos periódicos, foram: ano, espécie, estado, região, critérios estatísticos utilizados, modelos de volume avaliados, melhor modelo, método de cubagem rigorosa e valores do coeficiente de determinação e erro padrão da estimativa.

\section{Situação geral}

A revisão bibliográfica demonstrou que, dentre 40 artigos consultados (Tabela 1), o periódico Pesquisa Florestal Brasileira (PFB) e a Revista Árvore detém a maior quantidade de publicações acerca de equações volumétricas (Paula Neto 1977; Oliveira et al. 2005; Machado et al. 2008; Tonini et al. 2009; Soares et al. 2011; Santos et al. 2012; Melo et al. 2013, Barreto et al. 2014). 
Tabela 1. Periódicos, quantidade absoluta e quantidade relativa de trabalhos sobre volume de árvores no Brasil.

\begin{tabular}{lll}
\hline Revistas & Quant. & $\%$ \\
\hline Pesquisa Florestal Brasileira & 6 & 15,0 \\
Revista Árvore & 6 & 15,0 \\
Revista Floresta & 4 & 10,0 \\
Scientia Forestalis & 4 & 10,0 \\
Revista Biosfera & 3 & 7,5 \\
Revista Cerne & 3 & 7,5 \\
Acta Amazônica & 3 & 7,5 \\
Floram & 1 & 2,5 \\
Outras & 10 & 25,0 \\
\hline Total & 40 & 100 \\
\hline
\end{tabular}

A Revista Floresta e Scientia Forestalis, destacaram-se como os periódicos em segundo lugar em trabalhos sobre volumetria de árvores (Guimarães e Leite 1996; Rezende et al. 2006; Lima et al. 2014; Gouveia et al. 2015; Sales 2015; Cysneiros et al. 2017). Já, a Revista Floresta foi a primeira a publicar artigos acerca do assunto no início da década de 1970 (Hosokawa e Kajiya 1971; Veiga 1973; Siqueira 1977; Higuchi et al. 1979; Machado 1979; Silva e Schineider 1979).

Na Figura 1, foi possível verificar o desenvolvimento das publicações nas décadas de 1970, 1980, 1990, de 2000 a 2009 e de 2010 a 2018, que correspondem à 10\%,6\%,7\%,19\% e $58 \%$, respectivamente. A partir do ano 2000 , nota-se uma expressiva intensificação dos estudos sobre volumetria no Brasil, onde, em 18 anos se concentraram $77 \%$ das publicações.

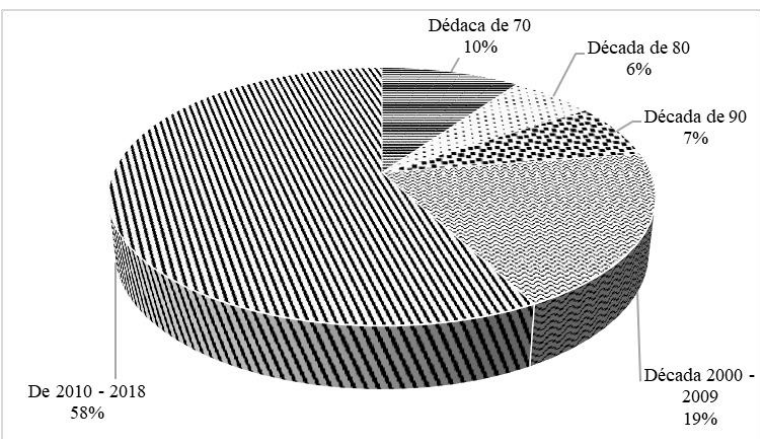

Figura 1. Porcentagem dos artigos científicos sobre equação volumétrica realizados em diferentes períodos "década" para povoamentos florestais brasileiros.

\section{Povoamento florestal estudado}

Dos artigos analisados, observou-se que a maioria dos trabalhos utilizaram dados do gênero Eucalyptus (Figura 2), onde as espécies mais estudadas foram: Eucalyptus grandis Hill ex Maiden, Eucalyptus uropylla S.T. Blake e o hibrido Eucalyptus urograndis, cada um com $21,43 \%$ dos artigos. O gênero Pinus, também apresentou uma parcela significativa dos estudos feitos, com destaque para o Pinus Taeda Lambert $(50 \%)$.

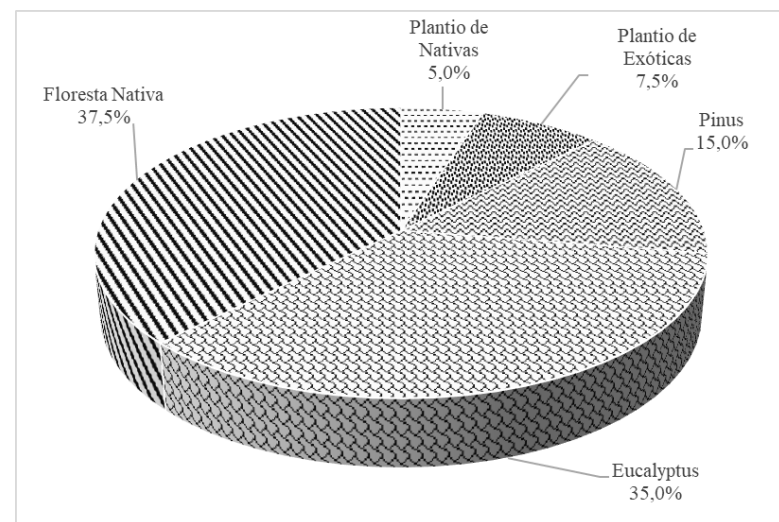

Figura 2. Tipos de povoamentos florestais brasileiros onde se realizaram estudos sobre volumetria.

Plantações de espécies nativas, como Carapa guianensis Aubl., Swietenia macrophylla King, Bertholletia excelsa Bonpl., Tabebuia avellanedae Lorentz ex Griseb e Hymenaea courbaril L. apresentaram representatividade de 5,0\%. Já, trabalhos feitos em plantios com espécies exóticas diferentes do eucalipto e pinho, representaram quase $7,5 \%$. Por fim, $37,5 \%$ dos artigos científicos correspondem aos trabalhos conduzidos em florestas nativas brasileiras.

O cenário caracterizado quanto ao povoamento florestal brasileiro, pode estar relacionado ao fato de que, segundo o IBÁ (2017), a área de plantios florestais totalizou 7,84 milhões de hectares em 2016. Destaque é dado ao gênero Eucalyptus, com cerca de 5,67 milhões de hectares (72,3\%), principalmente, nos Estados de Minas Gerais (24\%), São Paulo (17\%) e Mato Grosso do Sul (15\%). Já, os plantios com o Gênero Pinus, ocupam cerca 1,6 milhão de hectares se concentrando nos estados do Paraná (42\%) e em Santa Catarina (34\%). Portanto, devido a maior participação desses dois gêneros $(51,22 \%)$, exige-se maior gama de informações e pesquisas afins para definir-se os modelos volumétricos mais apropriados aos plantios dos gêneros Eucalyptus e Pinus.

\section{Estados da Federação e Regiões da fonte de dados}

Com relação à fonte de dados dos artigos científicos (Figura 3), 52,5\% estão concentrados em 4 estados brasileiros, quais sejam: Minas Gerais, Pará, Mato Grosso e Bahia. Outros 11 estados e o Distrito Federal, fornecem dados para 47,5\% dos artigos, que são: Amazonas, Amapá, Espirito Santo, Paraná, Pernambuco, Rio Grande do Sul, Rondônia, Roraima, São Paulo e Sergipe.

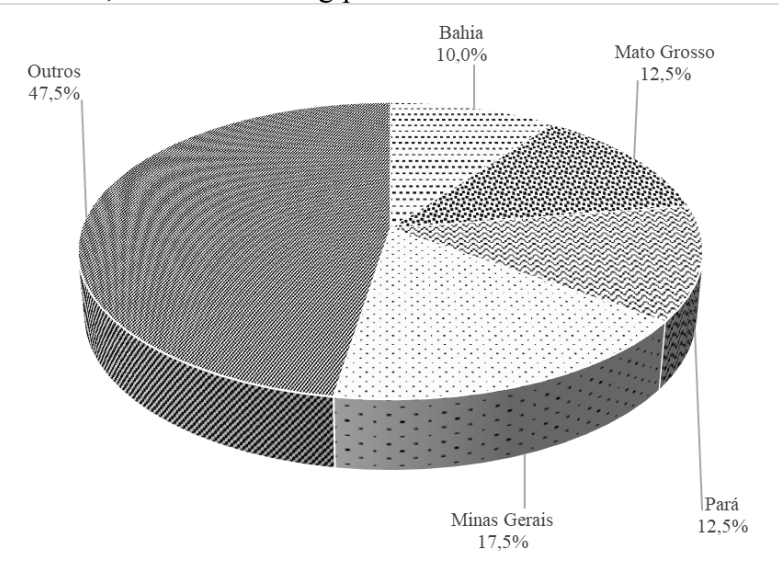

Figura 3. Porcentagem da fonte de dados dos artigos científicos sobre volumetria por estado brasileiro. 
Diante disso, evidencia-se uma participação equilibrada das regiões Sudeste, Centro-Oeste, Norte e Nordeste (Figura 4). Tal cenário, provavelmente, explica-se pelo fato da maior área de plantios florestais se localizarem nos estados brasileiros de Minas Gerais, Paraná, São Paulo, Mato Grosso do Sul e Bahia (IBÁ 2017), pois, quatro desses estados localizam-se nas regiões que mais fornecem dados para estudos de volumetria.

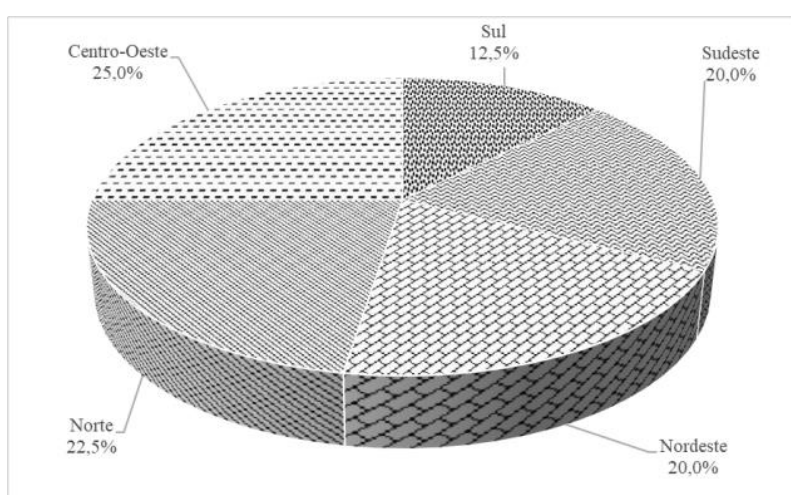

Figura 4. Porcentagem dos artigos científicos sobre volumetria por região brasileira.

Com relação à Região Norte, apesar de não apresentar grande destaque com relação às florestas plantadas, $78 \%$ das fontes de dados para ajuste de equações volumétricas são advindos de florestas nativas, por vezes, utilizadas para apoiar o manejo florestal desenvolvido na área (Barreto et al. 2014; Lima et al. 2014; Oliveira et al. 2017; Fernandes et al. 2017).

\section{Número de árvores cubadas e método de obtenção do volume real}

O número de árvores cubadas, geralmente, está relacionado à variabilidade do povoamento e precisão desejada nas estimativas do volume. Nos estudos analisados, foram encontradas de 20 (Lima et al. 2014) a 200 (Lundgren et al. 2015; Fernandes et al. 2017) árvores cubadas, com média de 120 indivíduos. Número superior que 50 a 100 árvores considerado suficiente para cobrir toda a variação de diâmetro e altura das árvores de determinado povoamento florestal (Scolforo 1993; Guimarães e Leite 1996).

Pôde-se verificar que para o gênero Eucalyptus, o valor médio de 183 árvores cubadas e, para gênero Pinus, de 105 árvores cubadas, conforme: Campos et al. (1985), Couto e Bastos (1987), Batista et al. (2004), Machado et al. (2008), Colpini et al. (2009), Fraga et al. (2014), Miranda et al. (2014), Gimenez et al. (2015), Martins et al. (2015), Sales et al. (2015), Stepka et al. (2017), Andrade (2017) e Fernandes et al. (2017).

Apesar da fórmula de Smalian apresentar menor precisão em relação às demais (Machado et al. 2006), pôde-se verificar que esta fórmula é a mais utilizada, sendo encontrada em cerca de 86,49\% dos trabalhos (Couto e Bastos 1987; Batista et al. 2004; Thiersch et al. 2006; Colpini et al. 2009; Gouveia et al. 2015, Cysneiros et al. 2017, Gama et al. 2017, Lanssanova et al. 2018, dentre muitos outros).

Por outro lado, a Fórmula de Huber, foi encontrada em 5,41\% dos estudos (Rufini et al. 2010; Miguel et al. 2010), já, a Fórmula de Newton, foi utilizada em 2,70\% (Miguel et al. 2014). Tais resultados, decorrem da fórmula de Smalian ter mais praticidade na coleta dos dados que Huber e Newton, conforme ressaltam Sanquetta et al. (2011), Ferreira et al. (2011) e Fraga et al. (2014).

Nas publicações analisadas foram encontradas seções do tronco com medições, sem a cepa, nas posições 0,$1 ; 0,7 ; 1,3$ $\mathrm{m}$; e após essa posição, de metro e metro até a altura total
(Tonini et al. 2009); medições, sem cepa, nas posições 0,1 ; 0,$3 ; 0,5 ; 0,7 ; 0,9 ; 1,2 ; 1,3 ; 1,5 ; 1,8 ; 2,1 ; 2,4 ; 2,7 ; 3,0 ; 5,0 ; 5,2$; $7,0 \mathrm{~m}$ e após essa posição a cada $2 \mathrm{~m}$ até $4 \mathrm{~cm}$ de diâmetro, (Rocha et al., 2010); medições, com cepa, nas posições 0,0 ; 0,$3 ; 1,1 \mathrm{~m}$ e a partir daí, de $1,1 \mathrm{em} 1,1$ metros até a altura total (Gama et al. 2017).

Durante a cubagem, a praticidade operacional em campo é o fator determinante, comprovado pela maciça utilização da Fórmula de Smalian. O seccionamento da cubagem também deve ser observado, pois, de acordo com Ribeiro et al. (2014), volumes obtidos com diferentes seccionamentos foram estatisticamente iguais. Desse modo, existe a possibilidade da adequação do seccionamento para as condições específicas de coleta dos dados de cubagem.

Critérios estatísticos adotados na seleção de equações volumétricas

A escolha de equações volumétricas é uma fase importante no inventário florestal, já que qualquer erro de tendência na estimativa do volume por árvore terá reflexos na estimativa do volume da população, causando a sub ou superestimação do volume (Campos et al. 1985; Guimarães e Leite 1996). Portanto, o principal objetivo de testar vários modelos estatísticos, é para definir o qual melhor modelo explica-se ao evento estudado com baixa possibilidade de erro.

Com relação às bibliografias analisadas, os critérios estatísticos mais utilizados são: coeficiente de determinação ajustado (em $80 \%$ dos trabalhos), erro padrão residual absoluto (58\%), erro padrão residual em porcentagem (43\%), erro padrão residual absoluto e em porcentagem (15\%), análise gráfica dos resíduos $(70 \%)$. Outros critérios, também foram encontrados, como: valor F $(23,33 \%)$, coeficiente de variação $(20 \%)$, significância dos parâmetros $(10 \%)$, raiz quadrada do erro médio em porcentagem (3,33\%), Critério de Informação de Akaike (3,33\%) e Critério de Informação Bayesiano (3,33\%).

Quanto ao modelo com melhor ajuste, o coeficiente de determinação ajustado (CDA) variou de 0,820 a 0,997 e o erro padrão residual (EPR) variou de $\pm 1,16 \% \mathrm{a} \pm 35,97 \%$. Obtevese, com estes resultados, o comportamento normal destas estatísticas sendo, em média, de 0,949 para CDA e de $\pm 14,51 \%$ para o EPR.

Detalhando-se as informações de Eucalipto, encontrou-se CDA de 0,820 a 0,991 e EPR de $\pm 6,50$ a $\pm 8,86 \%$. Isso resulta, em média, um $\mathrm{CDA}=0,965$ e $\mathrm{EPR}= \pm 7,75 \%$. Quanto ao gênero Pinus, o CDA variou de 0,947 a 0,997 e EPR de $\pm 9,90$ a $\pm 13,40 \%$, em média, resultou $\mathrm{CDA}=0,978$ e $\mathrm{EPR}=$ $\pm 11,31 \%$. Já, nas espécies nativas plantadas, o CDA variou de 0,950 a 0,980 e EPR de $\pm 2,90$ a $\pm 7,20 \%$, em média, resultou $\mathrm{CDA}=0,965$ e EPR $= \pm 5,05 \%$. Por fim em áreas florestais nativas, o CDA ficou entre 0,675 e 0,994 e EPR entre $\pm 1,16$ e $\pm 35,97 \%$, em média, o resultado foi $\mathrm{CDA}=0,926$ e EPR $=$ $\pm 18,18 \%$. Portanto, de acordo com estes resultados, os critérios de avaliação foram melhores para áreas plantadas quando comparado aos resultados encontrados para áreas florestais nativas.

\section{Modelos volumétricos mais difundidos}

A utilização de modelos volumétricos depende da análise dos resultados. São selecionados modelos lineares e não lineares, de simples entrada ou mais, de forma a gerar equações que aumentem as possibilidades de sucesso na estimativa desejada.

Dentre os trabalhos analisados, foram avaliados mais de 40 modelos volumétricos diferentes, sendo os dez mais usuais: Spurr, presente em $65 \%$ dos artigos consultados, Schumacher - Hall logarítmico em 55\%, Spurr logarítmico em 45\%, Stoate e Kopezky-Gehrhardt em 35\%, Naslund e 
Husch em 30\%, Hohenadl - Krenn em 27\%, Brenac em $22,50 \%$ e o modelo de Schumacher - Hall em 20\%.

Apesar de existirem diversos modelos de volume, alguns destacam-se por obterem o melhor ajuste, como o de Schumacher e Hall e o de Spurr. Dentre estes, o modelo de Schumacher e Hall na forma logarítmica tem sido o mais difundido no Brasil por resultar em estimativas não tendenciosas (Rufini et al. 2010; Azevedo et al. 2011; Miranda et al. 2015)

Conforme a literatura revisada, $40 \%$ dos artigos científicos tiveram o modelo de Schumacher-Hall logarítmico como sendo o de melhor ajuste, $10 \%$, apresentaram um melhor ajuste para o modelo de Spurr, em 7,5\% o modelo de Spurr Logarítmico apresentou melhor ajuste, demonstrando a abrangência desses modelos pela qualidade e, ou, facilidade de ajuste. Os modelos de Takata, Brenac, Husch, Stoate, Naslund, Prodan e outros também foram observados como melhor ajuste nos artigos analisados. Assertiva corroborado pelos estudos conduzidos por Pelissari et al. (2011), Miguel e Leal (2012), Melo et al. (2013), Barreto et al. (2014), Miguel et al. (2014), Silvestre et al. (2014) e Andrade (2017).

$\mathrm{Na}$ literatura foi possível observar diferentes modelos recomendados para estimativa volumétrica. Com isso, ressalta-se a importância do teste de diferentes modelos para a prescrição da equação volumétrica adequada a um determinado cenário de estudo.

\section{Considerações Finais}

A maior parcela de publicações sobre volumetria é encontrada a partir do ano 2000, sendo que as revistas PFB, Árvore e Floresta, apresentaram o maior número de artigos, concentrando o total de $40 \%$ dos estudos sobre modelos volumétricos

A floresta nativa é alvo de $37,5 \%$ do total dos trabalhos analisados, seguido do plantio de eucalipto com $35 \%$. O pinus ficou com $15 \%$ dos trabalhos.

Em relação à origem dos dados nos artigos científicos, $52,5 \%$ estão concentrados em quatro (04) estados brasileiros, quais sejam: Minas Gerais, Pará, Mato Grosso e Bahia.

$\mathrm{Na}$ maior parte da bibliografia analisada, a Fórmula de Smalian foi utilizada para a realização da cubagem rigorosa, com $86,49 \%$ dos trabalhos.

Foram usados mais de 40 modelos volumétricos diferentes, sendo os seis mais usuais, os modelos de Spurr, Shumacher - Hall logarítmico, Spurr logarítmico, Stoate, Kopezky-Gehrhardt e Naslund. Dentre os modelos normalmente testados, o modelo de Shumacher - Hall logarítmico foi o modelo de melhor ajuste na maioria dos trabalhos, com $40 \%$.

Os critérios estatísticos, massivamente utilizados para avaliar a qualidade do ajuste dos modelos, são: coeficiente de determinação ajustado, erro padrão residual e análise gráfica dos resíduos.

\section{Referências}

Andrade EM (1961) O eucalipto. $2^{\text {a }}$ edição. Jundiaí: Cia Paulista de Estradas de Ferro. 681p.

Andrade VCL (2017) Modelos volumétricos de dupla entrada para aplicar em povoamentos florestais brasileiros. Floresta e Ambiente, 24 (2): ed. e00135415. doi: 10.1590/2179-8087.135415

Azevedo GB, Sousa GTO, Barreto PAB, Conceição-Júnior V (2011) Estimativas volumétricas em povoamentos de eucalipto sob regime de alto fuste e talhadia no sudoeste da Bahia. Pesquisa Florestal Brasileira, 31 (68): 309-318. doi: 10.4336/2011.pfb.31.68.309
Azevedo TL, Mello AA, Ferreira RA, Sanquetta CR, Nakajima NY (2011) Equações hipsométricas e volumétricas para um povoamento de Eucalyptus sp. localizado na FLONA do Ibura, Sergipe. Revista Brasileira de Ciências Agrárias, 6 (1): 105-112. doi: 10.5039/agraria.v6i1a861

Barreto WF, Leão FM, Menezes MC de, Souza DV (2014) Equação de volume para apoio ao manejo comunitário de empreendimento florestal em Anapu, Pará. Pesquisa Florestal Brasileira, 34 (80): 321-329. doi: 10.4336/2014.pfb.34.80.721

Batista JLF, Marquesini M, Viana VM (2004) Equações de volume para árvores de caxeta (Tabebuia cassinoides) no estado de São Paulo e sul do estado do Rio de Janeiro. Scientia Forestalis, 65: 162-175.

Campos JCC, Júnior TLT, Paula Neto F (1985) Ainda, sobre a seleção de equações de volume. Revista Árvore, 9 (2): 115-126.

Colpini C, Travagin DP, Soares TS, Silva VSM (2009) Determinação do volume, do fator de forma e da porcentagem de casca de árvores individuais em uma Floresta Ombrófila Aberta na região noroeste de Mato Grosso. Acta Amazonica, 39 (1): 97-104. doi: 10.1590/S0044-59672009000100010

Couto HD, Bastos NLM (1987) Modelos de equações de volume e relações hipsométricas para plantações de Eucalyptus no Estado de São Paulo. Revista IPEF, 37: $33-44$

Cysneiros VC, Pelissari AL, Machado AS, Figueiredo Filho A, Souza L (2017) Modelos genéricos e específicos para estimativa do volume comercial em uma floresta sob concessão na Amazônia. Scientia Forestalis, 45 (114): 295-304. doi: 10.18671/scifor.v45n114.06

Fernandes AMV, Gama JRV, Rode R, Melo LO (2017) Equações volumétricas para Carapa guianensis Aubl. e Swietenia macrophylla King em sistema silvipastoril na Amazônia. Nativa, 5 (1): 73-77. doi: 10.5935/23187670.v05n01a12

Ferreira JCS, Silva JAA, Miguel EP, Encinas JI, Tavares JA (2011) Eficiência relativa de modelos volumétricos com e sem a variável altura da árvore. Acta Tecnológica, 6 (1): 89-102.

Fraga MP, Barreto PAB, Paula A (2014) Estimação de volume de Pterogyne nitens em plantio puro no sudoeste da Bahia. Pesquisa Florestal Brasileira, 34 (79): 207-215. doi: 10.4336/2014.pfb.34.79.703

Gama JRV, Souza AL, Veira DS, Leite HG (2017) Equações de volume para uma floresta ombrófila aberta, município de Codó, estado do Maranhão. Revista Brasileira de Ciências Agrárias, 12 (4): 535542. doi: 10.5039/agraria.v12i4a5489

Gimenez BO, Danielli FE, Oliveira CKAD, Santos JD, Higuchi N (2015) Equações volumétricas para espécies comerciais madeireiras do sul do estado de Roraima. Scientia Forestalis, 43 (106): 291-301.

Gouveia JF, Silva JAA, Ferreira RLC, Gadelha FHL, Lima Filho LMA (2015) Modelos volumétricos mistos em clones de Eucalyptus no polo gesseiro do Araripe, Pernambuco. Revista Floresta, 45 (3): 587-598. doi: 10.5380/rf.v45i3.36844 
Guimarães DP, Leite HG (1996) Influência do número de árvores na determinação de equação volumétrica para Eucalyptus grandis. Scientia Forestalis, 50: 37-42.

Heinsdjick D, Soares RO, Andel S, Ascoly RB (1965) Plantações de eucalipto no Brasil: estudo preliminar dos volumes e capacidade de produção. Rio de Janeiro: Ministério da Agricultura, Departamento de Recursos Naturais Renováveis. 69 p. (Boletim n ${ }^{\circ} 10$ ).

Higuchi N, Gomes B, Santos J, Constantino NA (1979) Tabela de volume para povoamento de Eucalyptus grandis plantados no munícipio de Várzea Grande (MT). Revista Floresta, 10 (1): 43-47. doi: 10.5380/rf.v10i1.6232

Hosokawa RT, Kajiya S (1971) Ensaio da aplicabilidade da fórmula franco-inglesa como modelo matemático para a volumetria dos produtos de desbastes da Araucaria angustifolia para a indústria papeleira. Revista Floresta, 3 (2): 75-82. doi: 10.5380/rf.v3i2.5722

INDÚSTRIA BRASILEIRA DE ÁRVORES - IBÁ (2017) Relatório 2017. 80p. http://iba.org/images/shared/Biblioteca/IBA_Relatorio Anual2017.pdf

Lanssanova LR, Silva FA, Schons CT, Pereira ACS (2018) Comparação entre diferentes métodos para estimativa volumétrica de espécies comerciais da Amazônia. BIOFIX Scientific Journal, 3 (1): 109-115. doi: 10.5380/biofix.v3i1.57489

Leite HG, Andrade VCL (2002) Um método para condução de inventários florestais sem o uso de equações volumétricas. Revista Árvore, 26 (3): 321328. doi: 10.1590/S0100-67622002000300007

Lima RB, Aparício OS, Ferreira RLC, Silva WC, Guedes MC, Oliveira CP, Silva DMS, Batista APB (2014) Volumetria e classificação da capacidade produtiva para Mora paraenses (Ducke) no estuário amapaense. Scientia Forestalis, 42 (101): 141-154.

Lundgren WJC, Silva JAA, Ferreira RLC (2015) Estimação do volume de madeira de eucalipto por cokrigagem, krigagem e regressão. Cerne, 21 (2): 243 250. doi: $10.1590 / 01047760201521021532$

Machado AS (1979) Tabela de volume para Pinus taeda na região de Telêmaco Borba, PR. Revista Floresta, 10 (1): 29-35. doi: 10.5380/rf.v10i1.6236

Machado SA, Téo SJ, Urbano E, Figura M A, Silva LCR (2006) Comparação de métodos de cubagem absolutos com o volume obtido pelo xilômetro para bracatinga (Mimosa scabrella Bentham). Cerne, 12 (3): 239-253.

Machado AS, Figura MA, Silva LCR, Téo SJ, Stolle L, Urbano E (2008) Modelagem volumétrica para bracatinga (Mimosa scabrella) em povoamentos da Região Metropolitana de Curitiba. Pesquisa Florestal Brasileira, 56: 17-29.

Martins RM, Leite MVS, Cabacinha CD, Assis AL (2015) Teste de identidade de modelos volumétricos para povoamentos de Eucalyptus sp. em sete municípios de Minas Gerais. Enciclopédia Biosfera, 11 (21): 18181833.

Melo LC, Barreto PAB, Oliveira FGRB, Novaes AB (2013) Estimativas volumétricas em povoamento de Pinus caribaea var. hondurensis no Sudoeste da Bahia.
Pesquisa Florestal Brasileira, 33 (76): 379-386. doi: 10.4336/2013.pfb.33.76.459

Miguel EP, Canzi LF, Rufino RF, Santos GA (2010) Ajuste de modelo volumétrico e desenvolvimento de fator de forma para plantios de Eucalyptus grandis localizados no município de Rio Verde - GO. Enciclopédia Biosfera, 6 (11): 1-13.

Miguel EP, Leal FA (2012) Seleção de equações volumétricas para a predição do volume total de Eucalyptus urophylla $\mathrm{S}$. T. Blake na região norte do estado de Goiás. Enciclopédia Biosfera, 8 (14): 1372 1386.

Miguel EP, Leal FA, Ono HA, Leal UAS (2014) Modelagem na predição do volume individual em plantio de Eucalyptus urograndis. Revista Brasileira de Biometria, 32 (4): 584-598.

Miranda DLC, Paro BAV, Costa GR (2014) Estimativa do volume em árvores de Hymenaea coubaril e Trattinnickia burserifolia no norte de Mato Grosso. Nativa, 2 (4): 219-223. doi: 10.14583/23187670.v02n04a06

Miranda DLC, Junior VB, Gouveia DM (2015) Fator de forma e equações de volume para estimativa volumétrica de árvores em plantio de Eucalyptus urograndis. Scientia Plena, 11 (3): 1-8.

Oliveira MLR, Soares CPB, Souza AL, Leite HG (2005) Equações de volume de povoamento para fragmentos florestais naturais do município de Viçosa, Minas Gerais. Revista Árvore, 29 (2): 213-225. doi: 10.1590/S0100-67622005000200005

Oliveira RCA, Rode R, Gama JRV, Almeida EC (2017) Equações volumétricas para Couratari stellata A.C Smith (Tauari) na Floresta Nacional do Tapajós. Nativa, 5 (2): 138-144.

Paula Neto F (1977) Tabelas volumétricas com e sem casca para Eucalyptus saligna. Revista Árvore, 1 (1): $31-54$

Paula Neto F, Souza AL, Quintaes PCG, Soares VP (1983) Análise de equações volumétricas para Eucalyptus spp., segundo o método de regeneração na região de José de Melo. Revista Árvore, 7 (1): 56-70.

Pelissari AL, Lanssanova LR, Drescher R (2011) Modelos volumétricos para Pinus tropicais em povoamento homogêneo, no Estado de Rondônia. Pesquisa Florestal Brasileira, 31 (67): 173-181. doi: 10.4336/2011.pfb.31.67.173

Rezende AV, Vale AT, Saquetta CR, Figueiredo Filho A, Felfili JA (2006) Comparação de modelos matemáticos para estimativa do volume, biomassa e estoque de carbono da vegetação lenhosa de um cerrado sensu stricto em Brasília, DF. Scientia Forestalis, 71: 65-76.

Ribeiro RB, Gama JRV, Melo LO (2014) Seccionamento para cubagem e escolha de equações de volume para floresta nacional do Tapajós. Cerne, 20 (4): 605-612. doi: 10.1590/01047760201420041400

Rocha TB, Cabacinha CD, Almeida RC, Paula A, Santos RC (2010) Avaliação de métodos de estimativa de volume para um povoamento de Eucalyptus urophylla S. T. Blake no Planalto da Conquista - BA. Enciclopédia Biosfera, 6 (10): 1-13. 
Rufini AL, Scolforo JRS, Oliveira AD, Mello JM (2010) Equações volumétricas para o cerrado sensu stricto, em Minas Gerais. Cerne, 16 (1): 1-11. doi: 10.1590/S0104-77602010000100001

Sales FCV, Silva JAA, Ferreira RLC, Gadelha FHL (2015) Ajustes de modelos volumétricos para o clone Eucalyptus grandis $\mathrm{x}$ E. urophylla cultivados no agreste de Pernambuco. Revista Floresta, 45 (4): 663670. doi: 10.5380/rf.v45i4.37594

Santos AT, Mattos PP, Braz EM, Rosot NC (2012) Equação de volume e relação hipsométrica para plantio de Ocotea porosa. Pesquisa Florestal Brasileira, 32 (69): 13-21. doi: 10.4336/2012.pfb.32.69.13

Scolforo JRS (1993) Mensuração Florestal 3: Relação Quantitativas em Volume, Peso e a Relação Hipsométrica. Lavras: ESAL/FAEPE. 292p.

Silva JNM, Schneider PB (1979) Comparação de equações de volume para povoamento de Acacia mearsii de Wild (acácia negra) no estado do Rio Grande do Sul. Revista Floresta, 10 (1): 36-42.

Silvestre R, Bonazza M, Stang M, Lima GCP, Koepsel DA, Marco FT, Ciarnoschi LD, Scariot S, Morês DF (2014) Equações volumétricas em povoamentos de Pinus taeda L. no município de Lages - SC. Nativa, 2 (1): 1-5. doi: 10.14583/2318-7670.v02n01a01

Siqueira JDP (1977) Tabelas de volume para povoamentos nativos de Araucária angustifolia (Bert.) O. Ktze., no sul do Brasil. Revista Floresta, 8 (1): 7-12. doi: 10.5380/rf.v8i1.5814

Soares CPB, Martins FB, Leite Junior HU, Silva G, Figueiredo L (2011) Equações hipsométricas, volumétricas e de taper para onze espécies nativas. Revista Árvore, 35 (5): 1039-1051.

Spurr SH (1952) Forest inventory. New York: The Ronald Press Company. 476p.

Stepka TF, Zeny Júnior GA, Lisboa GS, Cerqueira CL Pesck VA, Roik M (2017) Modelos volumétricos e funções de afilamento para Pinus taeda L. na região dos Campos Gerais, Paraná, Brasil. Revista Espacios, 38 (21): 26-35.

Thiersch CR, Scolforo JR, Oliveira AD, Rezende GDSP, Maestri R (2006) O uso de modelos matemáticos na estimativa da densidade básica da madeira em plantios de clones de Eucalyptus sp. Cerne, 12 (3): 264-278.

Thomas C, Andrade CM, Schneider PR, Finger CAG (2006) Comparação de equações volumétricas ajustadas com dados de cubagem e análise de tronco. Ciência Florestal, 16 (3): 319-327. doi: 10.5902/198050981911

Tonini H, Costa MCG, Schwengber LAM (2009) Crescimento da Teca (Tectona grandis) em reflorestamento na Amazônia Setentrional. Pesquisa Florestal Brasileira, 59: 5-14, 2009. Doi: 10.4336/2009.pfb.59.05

Veiga RAA (1972) Equações volumétricas para Eucalyptus saligna Smith, em ocasião de primeiro corte. Botucatu: F.C.M.B.B. 172p.

Veiga RAA (1973) Comparação de equações de volume para Eucalyptus saligna Smith III - Equações logarítmicas formais e não formais. Revista Floresta, 4 (3): 5-14. 10.5380/rf.v4i3.5768 